OPEN ACCESS

Edited by:

Maria Cristina Mele,

Catholic University of the Sacred

Heart, Italy

Reviewed by:

Maria Montserrat Diaz Pedrosa,

State University of Maringá, Brazil

Alessandro Laviano,

Sapienza University of Rome, Italy

*Correspondence:

Xinying Wang

wangxinying@nju.edu.cn

tThese authors have contributed equally to this work

Specialty section:

This article was submitted to Clinical Nutrition,

a section of the journal

Frontiers in Nutrition

Received: 31 March 2021

Accepted: 16 August 2021

Published: 03 September 2021

Citation:

Deng G, Lei Q, Gao X, Zhang Y,

Zheng $\mathrm{H}, \mathrm{Bi} J$ and Wang $X$ (2021)

Glucagon-Like Peptide-2 Modulates

Enteric Paneth Cells Immune

Response and Alleviates Gut

Inflammation During Intravenous Fluid

Infusion in Mice With a Central

Catheter. Front. Nutr. 8:688715.

doi: 10.3389/fnut.2021.688715

\section{Glucagon-Like Peptide-2 Modulates Enteric Paneth Cells Immune Response and Alleviates Gut Inflammation During Intravenous Fluid Infusion in Mice With a Central Catheter}

\author{
Guifang Deng ${ }^{1,2+}$, Qiucheng Lei $^{3+}$, Xuejin Gao ${ }^{1}$, Yupeng Zhang ${ }^{1}$, Huazhen Zheng ${ }^{4}$, \\ Jingcheng $\mathrm{Bi}^{5}$ and Xinying Wang ${ }^{1 *}$
}

\begin{abstract}
' Research Institute of General Surgery, Affiliated Jinling Hospital, Medical School of Nanjing University, Nanjing, China, ${ }^{2}$ Department of Clinical Nutrition, Union Shenzhen Hospital of Huazhong University of Science and Technology, Shenzhen, China, ${ }^{3}$ Department of Hepatopancreatic Surgery, The First People's Hospital of Foshan, Foshan, China, ${ }^{4}$ Department of Clinical Laboratory, The First People's Hospital of Foshan, Foshan, China, ${ }^{5}$ Department of General Surgery, Taizhou People's Hospital, Taizhou, China
\end{abstract}

Background: Glucagon-like peptide-2 (GLP-2) has protective effects on gastrointestinal functions. Our previous study found that GLP-2 could significantly reduce intestinal permeability and bacterial translocation in total parenteral nutrition (TPN) animal model. However, the effects of GLP-2 on the impairment of the intestinal Paneth cells immune function and gut inflammation during intravenous fluid infusion mainly consisted of nutritional materials is currently scattered.

Objective: The current study was aimed to investigate the efficacy of the GLP-2 in alleviating gut inflammation and modulating enteric Paneth cells immune response in parenterally fed mice and its underlying mechanisms.

Methods: Thirty-six male ICR mice underwent venous catheterization were divided into 3 groups: Chow, TPN, and TPN+GLP-2 groups. GLP-2 was administered intravenously at $60 \mu \mathrm{g} /$ day for 5 days. The small intestine tissue and serum samples were collected on the 7 th day.

Results: Compared with the TPN group, the expression of tight junction proteins occludin and claudin-1 were significantly increased in the TPN+GLP-2 group. In addition, the expression of lysozyme, sPLA2, insulin-like growth factor-1, and epithelial protection and repair genes were improved in the TPN+GLP-2 group. The levels of IL- 6 and TNF- $\alpha$ proteins and mRNAs in the ileum tissues were remarkably reduced in the TPN+GLP-2 group, while IL-10 protein and mRNA level were elevated in the TPN+GLP-2 group (all $p$ $<0.05)$. Moreover, the TPN+GLP-2 group has higher levels of serum endotoxin, D-lactic acid, and MPO than those of the TPN group. 
Conclusions: GLP-2 alleviated gut inflammation and improved enteric Paneth cells immune responses through intravenous fluid infusion, possibly by improving the functioning of epithelial protection and repair, and reducing mucosal inflammatory responses.

Keywords: glucagon-like peptide-2, parenteral nutrition, inflammation, Paneth cells, intestinal immunity, intravenous infusion

\section{INTRODUCTION}

Intravenous (IV) infusion is a method that uses the principle of atmospheric pressure and hydrostatic pressure to input a large number of sterile liquids, electrolytes and drugs into the body through the vein. Due to different injection sites, it can be divided into peripheral venous infusion and central venous infusion. IV infusion is an indispensable method in parenteral nutritional therapy, through which the nutrients needed of patients are supplied. Parenteral nutrition (PN) has been the standardized treatment of patients with acute and/or chronic intestinal failure $(1,2)$. Some diseases make patients unable to tolerate enteral nutrition and can only rely on parenteral nutrition support, such as short bowel syndrome, small bowel resection, Crohn's disease or intestinal ischemia related to thrombosis, volvulus and trauma, intestinal obstruction and radiation enteritis (3). TPN is also suitable for patients with malnutrition during critical illness (4). Reported, nearly 400,000 patients in the United States rely on parenteral nutrition to survive every year, including more than 1,000 infants with congenital intestinal atresia $(5,6)$. Despite the apparent benefits, patients receiving $\mathrm{PN}$ via $\mathrm{IV}$ infusion are more likely to develop severe or fatal complications such as liver injury, intestinal atrophy, and immune function suppression $(1,2)$. Other impairments that may occur during PN via IV infusion include damage to the intestinal mucosal barrier, intestinal inflammatory response, increased cytokine release, and gut bacterial translocation (2).

Glucagon-like peptide-2 (GLP-2) is a 33-amino-acid proglucagon-derived peptide (7). It is produced by the enteroendocrine $\mathrm{L}$ cells in the intestinal epithelium, which is primarily located in the terminal ileum and proximal colon (7). Some research indicated that GLP-2 can promote crypt cell proliferation and mucosal healing, increase intestinal blood flow, improve gut barrier function, and exert an antiinflammatory effect (7-9). It has shown good therapeutic potential in experimental models after receiving total parenteral nutrition after partial bowel resection. In our previous study, we found that GLP-2 could significantly reduce intestinal permeability and bacterial translocation during parenteral

Abbreviations: PN, parenteral nutrition; TPN, total parenteral nutrition; GLP2, Glucagon-like peptide-2; ZO-1, zonula occludens-1; Tf1, trefoil factor-1; Tgfb1, transforming growth factor-b1; Egfr, epidermal growth factor receptor; Hgf, hepatocyte growth factor; Fgf7, keratinocyte growth factor; Igf1, insulin like growth factor-1; sPLA2, secretory phospholipase A2 proteins; GH, Growth hormone. nutrition (10), exhibiting its potential protective role on the intestinal barrier in total parenteral nutrition (TPN). Although some studies have provided insights into the protective effects of GLP-2 on short bowel syndrome and bowel injury (11-13), the available information regarding the effects of GLP-2 on the impairment of the intestinal Paneth cells immune function and gut inflammation during TPN is currently scattered.

Therefore, we investigate the protective effects of GLP2 against intestinal immune function damage and gut inflammation during intravenous fluid infusion via a central catheter and its possible mechanisms.

\section{MATERIALS AND METHODS}

\section{Animals}

This study was approved by the animal ethics committee of Jinling hospital (Ethical approval number: 2018NZGKJ034). A total of 36 male (aged 6-8 weeks) ICR mice were provided by the experimental animal research center of Jinling hospital. The animals initially weighed $20-25 \mathrm{~g}$ and were housed 12:12h light/dark cycle under suitable humidity and temperature conditions. Mice were freely accessed to water and food and with their activity unrestricted prior to the study (14). The mice were allowed to acclimatize to laboratory conditions for one week before the experimental protocol was initiated.

\section{Study Design}

Thirty-six male ICR mice were randomly divided into three groups: Chow (Control group, $n=12$ ), TPN (TPN + vehicle group, $n=12$ ), and TPN + GLP-2 (TPN + GLP-2 group, $n=12$ ). The Control group mice were fed with standard laboratory chow diet, while the other two experimental groups were respectively treated with $24 \mathrm{~h}$ continuous pumping of parenteral nutrition solution or parenteral nutrition combined with GLP-2 analog (teduglutide, PLLabs, Canada). GLP-2 therapy was provided intravenously at a dose of $60 \mu \mathrm{g} /$ day (30 $\mu \mathrm{g}$ intravenously, twice daily). This dose was selected because, in our previous studies, we found a positive effect of GLP-2 during TPN at the same dose (10).

\section{Animal Experiments}

The animal experiments were performed at a temperature of $24^{\circ} \mathrm{C}$ and a humidity of $40-50 \%$. Mice were anesthetized by intraperitoneal ketamine injection (100 mg/kg) and venous 

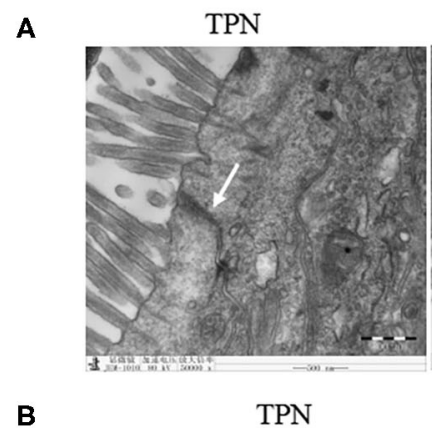

TPN+GLP-2

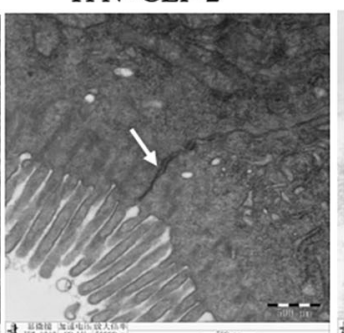

TPN+GLP-2

B
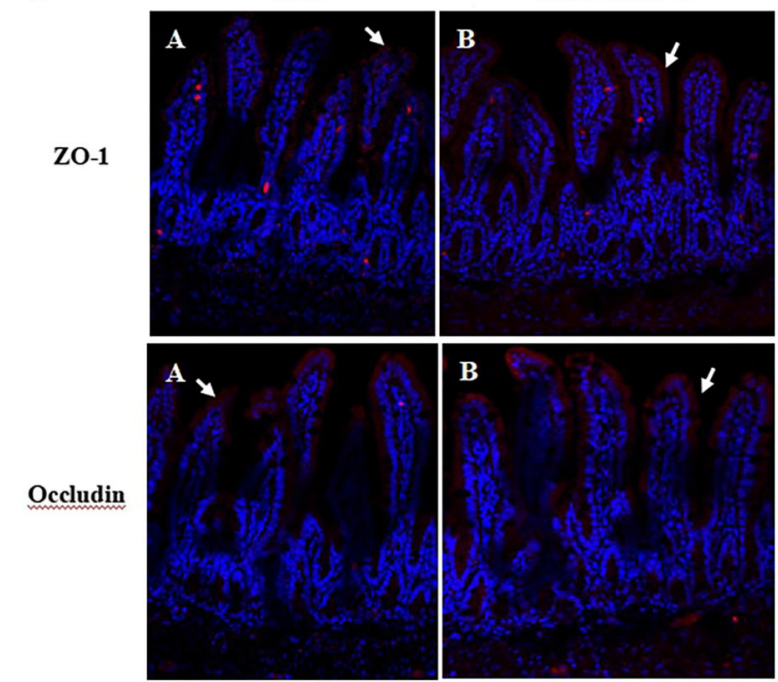

B
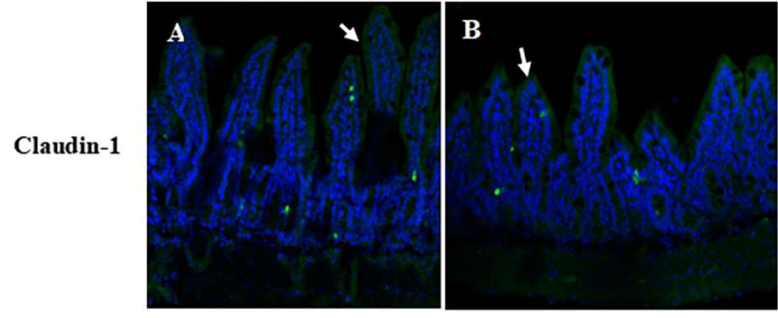

C
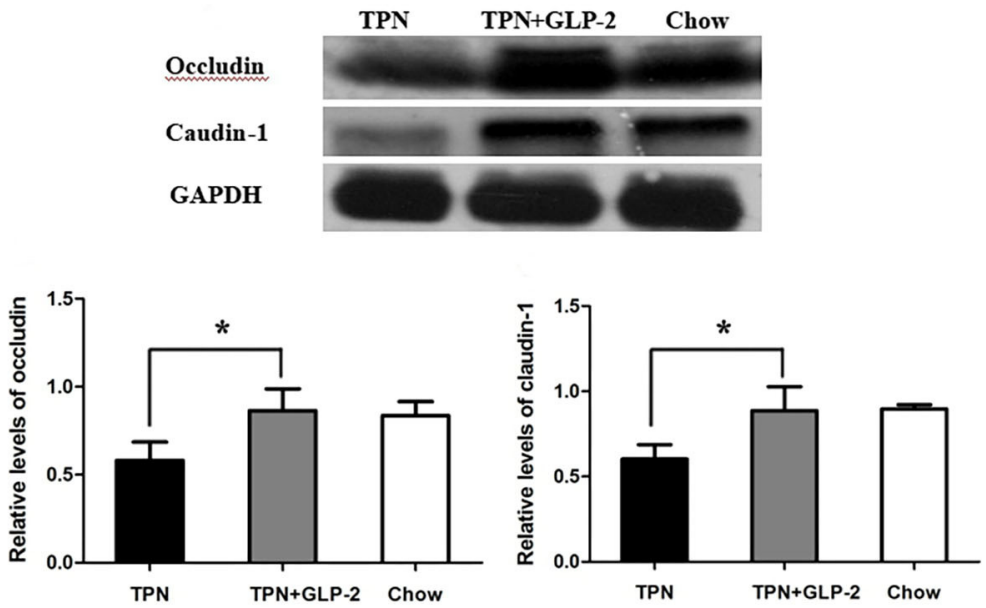

FIGURE 1 | The tight junctions and tight unction proteins. (A) Transmission electron microscopy. (B) Immunofluorescence. (C) Western blot analysis. Where *indicates $P<0.05$ for TNP vs. TPN+GLP-2 and Chow. 
A
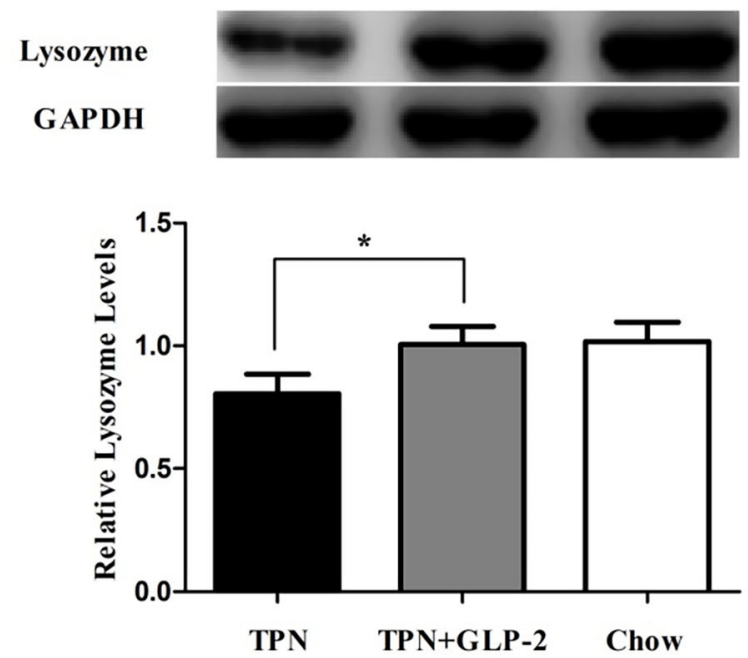

B

SPLA2

GAPDH

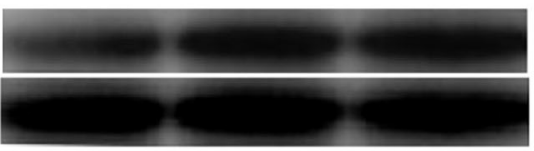

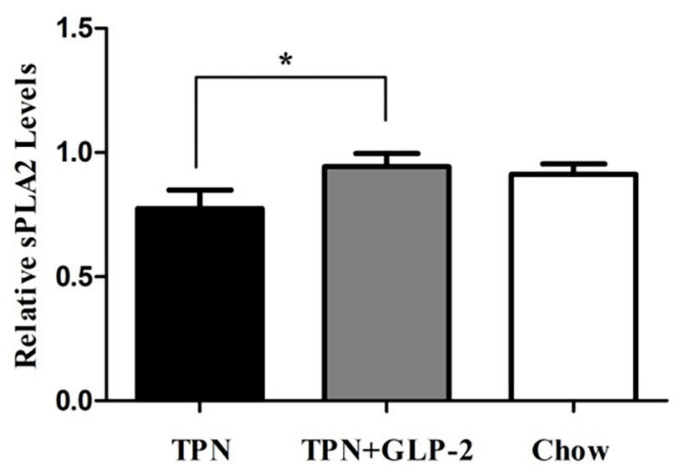

FIGURE 2 | The intestinal tissue Paneth cells function. (A) Lysozyme. (B) Secreted phospholipase A2. Where *indicates $p<0.05$ for TNP vs. TPN+GLP-2 and Chow.

catheter (Helix Medical Inc., Carpentaria, CA, USA) was surgically implanted in their right internal jugular vein, after which they were adapted to diet treatment for $24 \mathrm{~h}$ post-surgery (10). During the $48 \mathrm{~h}$ recovery period after surgery, the mice were treated with $0.9 \%$ saline through the catheter. Then mice received parenteral nutrition solution at rates of $4.4 \mathrm{~mL} /$ day 1 , $7.7 \mathrm{~mL} /$ day 2 , and $11 \mathrm{~mL} /$ day $3-5$ in the TPN and TPN+GLP-2 groups, as previously described (10). The Chow group mice were continuous intravenous pumping of saline via the right internal jugular vein, and had free access to water and Chow diet. Dissolve GLP-2 in sterile PBS and mice were intravenous injection 30 $\mu \mathrm{g}$ of GLP-2 (a total of $100 \mu \mathrm{L}$ of solution) twice daily for five days in the TPN+GLP-2 group. Mice in the Control and TPN group were also injected with the vehicle (100 $\mu \mathrm{L}$ of PBS) without GLP-2. The TPN solution provided approximately $1,951 \mathrm{~kJ} / \mathrm{kg}$ per day of energy and $16.4 \mathrm{~g} / \mathrm{kg}$ per day of protein. The PN formula mainly includes the following: 5.3\% free amino acids, $2.1 \%$ fat emulsion, $32 \%$ dextrose, multivitamins and electrolytes, with total calories $1,280 \mathrm{kcal} / \mathrm{L}$, and the non-protein calories and nitrogen ratio 149:1 (15). These values could meet the nutritional and energetic needs of mice weighing 25-30 g (10). In order to ensure that all three groups of mice receiving the same quantity of calories and proteins, each mouse is raised in a single cage. The control group calculated the amount of standard laboratory feeds every day.

Three groups of mice were anesthetized with $1 \%$ pentobarbital sodium $(30 \mathrm{mg} / \mathrm{kg})$ and then sacrificed after feeding for 5 days (i.e., 8 days after catheterization). We obtained portal vein blood samples and small intestine tissue. All the intestine tissue and serum samples were stored in the refrigerator at $-80^{\circ} \mathrm{C}$ for later testing.

\section{Transmission Electron Microscopy (TEM)}

A segment of the small intestine tissues approximately $0.5 \mathrm{~cm}$ in length from each sample was processed for examination under TEM.

\section{Immunofluorescence for Detection of Zonula Occludens-1 (ZO-1), Occludin, and Claudin-1 Tight Junction Proteins in the lleum Tissues}

The fixed ileum tissues were processed (Tissue-Tek VIP; Sakura Finetek Japan, Tokyo, Japan) and embedded in paraffin. The tissue sections were cut to a thickness of $5 \mu \mathrm{m}$ and deparaffinized. Antigen retrieval was performed by immersing sections in sodium citrate buffer $(\mathrm{pH}=6.0)$ at $95^{\circ} \mathrm{C}$ for $30 \mathrm{~min}$. The tissue sections were blocked in blocking buffer ( $5 \%$ normal goat serum, $5 \%$ bovine serum albumin, and $0.1 \%$ Tween 20 in sterile PBS) at $20-24^{\circ}$ Croom temperature, and then incubated with primary antibody at $4^{\circ} \mathrm{C}$. The tissue sections were washed three times in sterile PBS and incubated with secondary antibody at 20-24 Croom temperature for $60 \mathrm{~min}$ on the following day. Subsequently, after 3 washes with sterile PBS, the tissue sections were mounted in 50\% glycerol, photographed, and observed under a confocal microscope (Olympus, Tokyo, Japan). The evaluation procedure of acquired images were conducted blindly with the Image Pro Plus software. Rabbit anti-mouse occludin, claudin-1, and ZO-1 antibodies (Abcam, Cambridge, UK) at dilutions of 1:200 were then used for immunofluorescence (IF).

\section{Western Blotting}

The total concentrations of proteins in small intestinal tissues were determined using the bicinchoninic acid protein assay kit (Sangon Biotech Co., Shanghai, China). The protein concentration of each sample was $4 \mu \mathrm{g} / \mu \mathrm{L}$. The total protein $(50 \mu \mathrm{g})$ was electrophoresed on 8 or $10 \%$ sodium dodecyl sulfate polyacrylamide gel. The separated proteins were transferred to a PVDF membrane (Millipore Co., Billerica, MA, USA). Block the membrane for non-specific binding proteins in 5\% skimmed milk at room temperature $20-24^{\circ} \mathrm{C}$ for $1 \mathrm{~h}$, and then incubated overnight at $4^{\circ} \mathrm{C}$ with Anti-Lysozyme antibody (1:1,000; Abcam, Cambridge, MA, UK), Anti-occludin (1:1,000; Abcam, Cambridge, MA, UK), Anti-claudin-1(1:1,000; Abcam, 

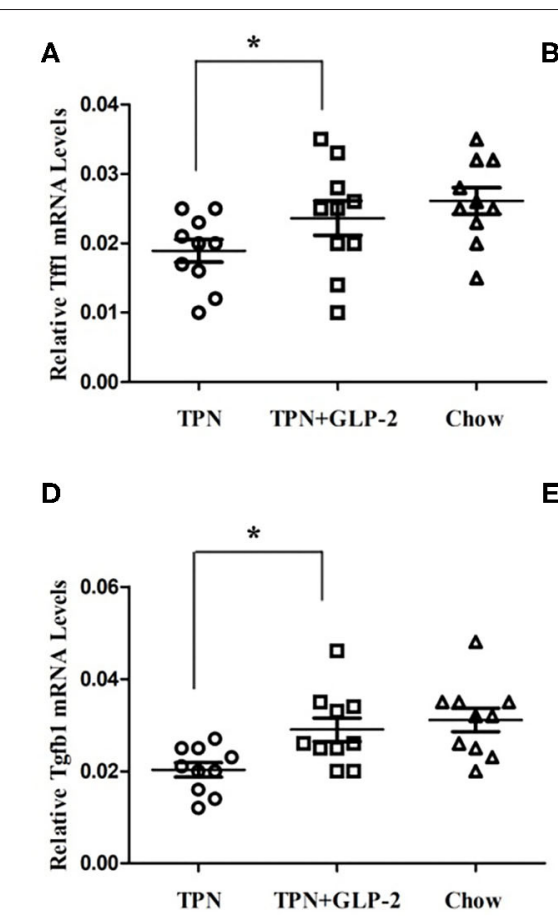

G

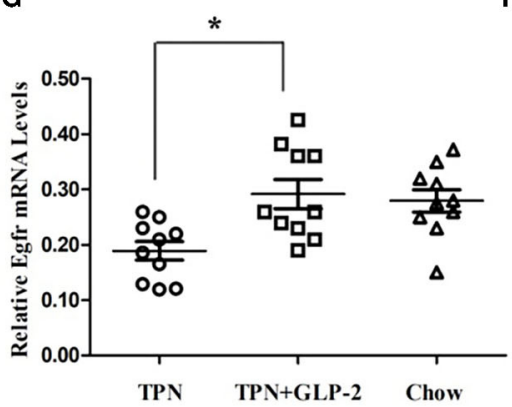

B

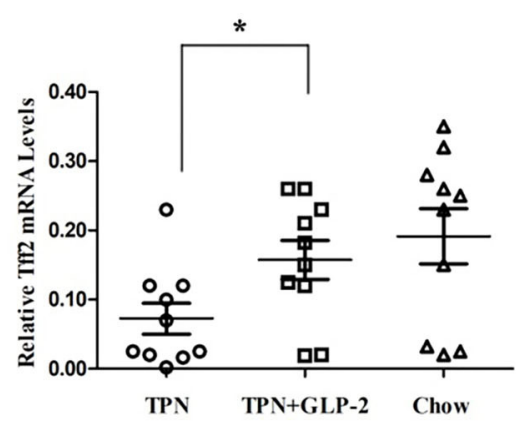

E

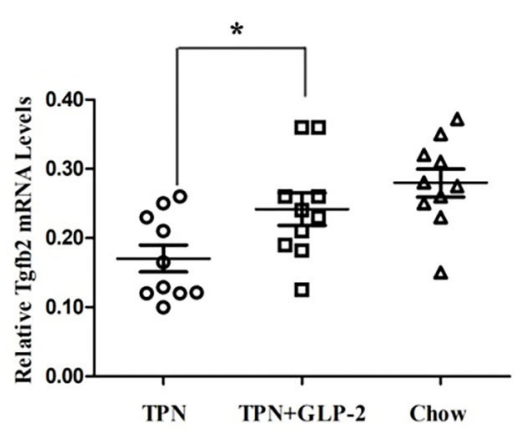

H

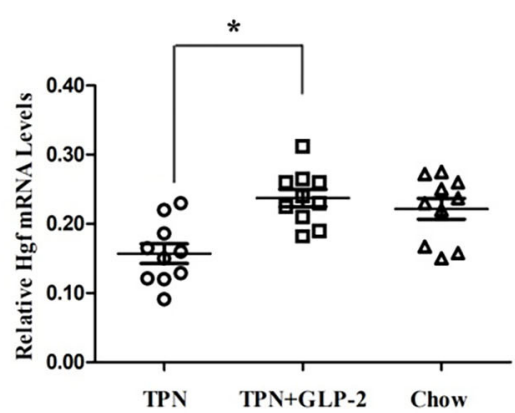

C

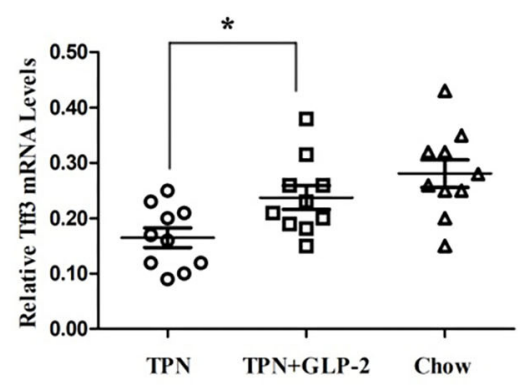

F

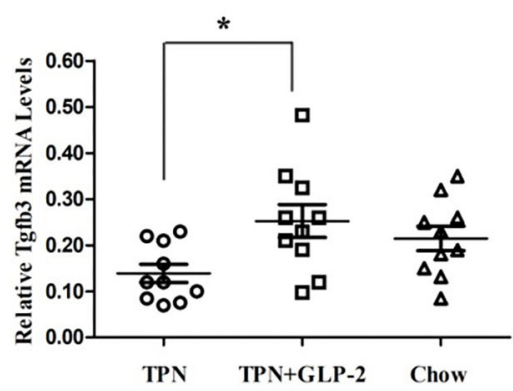

I

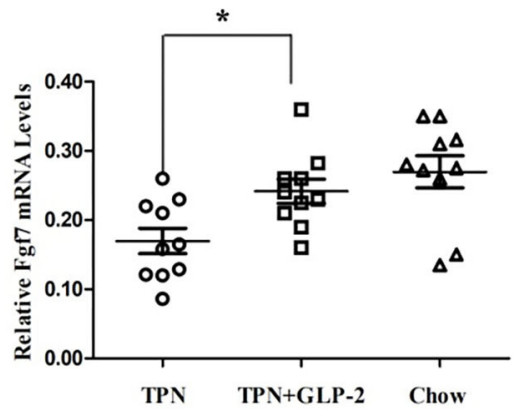

FIGURE 3 | The intestinal tissue the expression of epithelial protection and repair genes. (A-I) [trefoil factor-(Tff)1,2 and 3; transforming growth factor-(Tgf) b1,2 and 3; epidermal growth factor receptor (Egfr); hepatocyte growth factor (Hgf); keratinocyte growth factor (Fgf7)], where *indicates $p<0.05$ for TNP vs. TPN+GLP-2 and Chow.

Cambridge, MA, UK), Anti-secretory phospholipase A2 antibody rabbit (1:1,000; Abcam, Cambridge, MA, UK), and Anti-GAPDH antibody (1:10,000; Santa Cruz Biotechnology, MA, USA). Subsequently, the membrane was washed three or five times in Tris-buffered saline containing Tween-20 and incubated with appropriate species-specific secondary antibodies at $20-24^{\circ} \mathrm{C}$ for $1 \mathrm{~h}$ at room temperature. Use chemiluminescence detection reagents and expose them to Kodak XAR film. Using the GAPDH bands as an internal control, all band densities were analyzed with ImageJ software.

\section{Quantitative Real-Time PCR}

The expression levels of IL- 6 , IL-10, TNF- $\alpha$, trefoil factor- 1,2 and 3 (Tff-1,Tff-2, and Tff-3), transforming growth factor-b1, 2 and 3 (Tgfb-1,Tgfb-2, and Tgfb-3), epidermal growth factor receptor (Egfr), hepatocyte growth factor (Hgf), keratinocyte growth factor (Fgf7), and insulin like growth factor-1(Igf1) mRNA in the terminal ileum tissue were analyzed by qRT-PCR. The primers for gene sequences were provided by GenScript Co., Ltd. (Nanjing, China), and are listed in Supplementary Table 1. Use TRIzol reagent (TakaRa, Dalian, China) to extract total RNA from ileal tissue according to the instructions. Prime-Script RT kit (TaKaRa, Dalian, China) was used to reverse transcribed RNA into cDNA, according to the instructions. The $2^{-\Delta \Delta \mathrm{Ct}}$ method was used.

\section{Enzyme-Linked Immunosorbent Assay}

ELISA kits for mouse TNF- $\alpha$, mouse IL-6, mouse IL-10 (Nanjing BioSky Sci \& Tech Co., Ltd., Nanjing, China) and mouse insulin like growth factor-1 (Abcam, Cambridge, MA, UK) were used to determine the levels of TNF- $\alpha$, IL-6, IL-10, and Igf- 1 proteins, respectively, in small intestinal tissue homogenates, according to the manufacturer's instructions. 


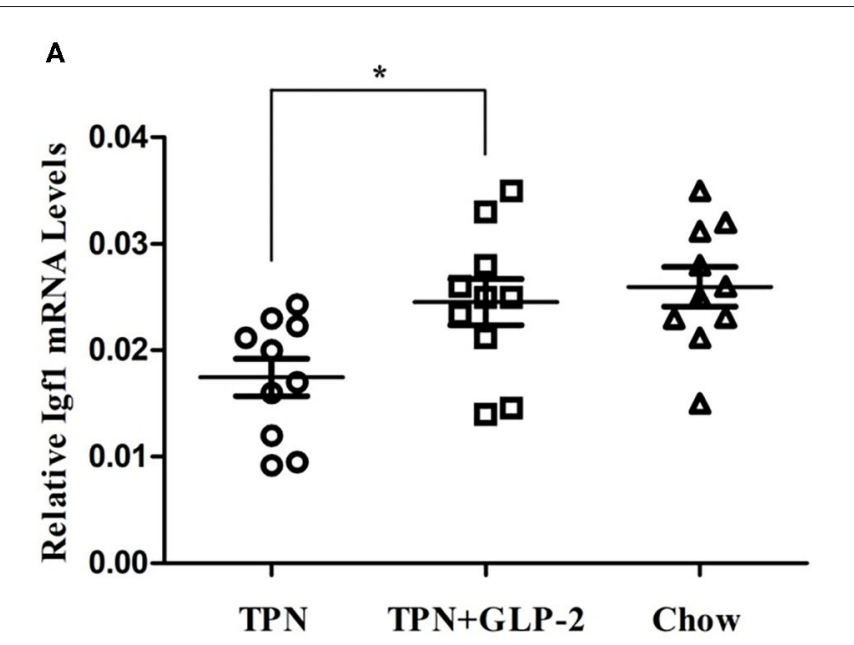

B

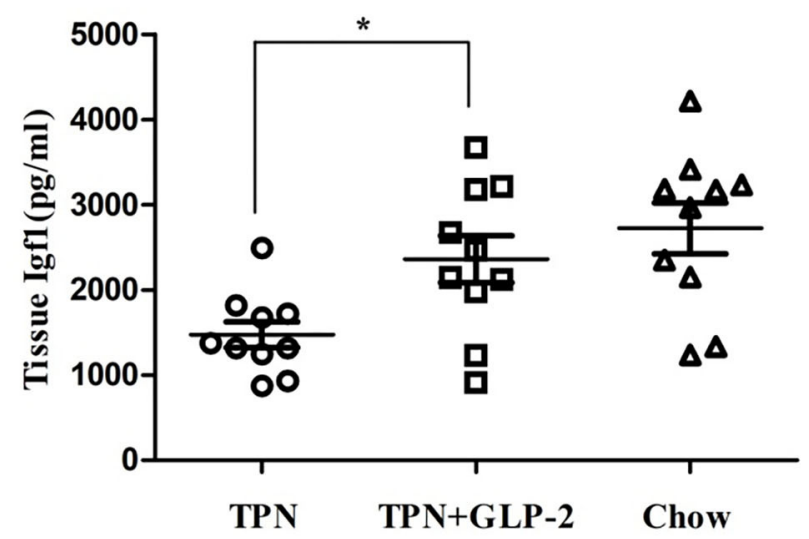

FIGURE 4 | The intestinal tissue the expression of insulin like growth factor-1. (A) Expression of insulin like growth factor-1 mRNA. (B) Expression of insulin like growth factor- 1 protein. Where *indicates $p<0.05$ for TNP vs. TPN+GLP-2 and Chow.

A mouse D-lactate ELISA kit (R\&D Systems Inc., Minneapolis, MN, USA) was also used to determine serum D-lactate levels, while following the manufacturer's instructions.

\section{Intestinal Tissue Myeloperoxidase (MPO) Activity Assay}

An MPO Activity Assay Kit (R\&D Systems Inc., Minneapolis, $\mathrm{MN}$, USA) was used to determine the MPO levels in small intestinal tissue homogenates, while following the manufacturer's instructions.

\section{Serum Endotoxin Activity Assay}

Serum endotoxin levels were determined using an Endotoxin Activity Assay Kit (EAATM, Spectral Diagnostics Inc., Xiamen, China), according to the manufacturer's instructions.

\section{Statistical Analyses}

Data were expressed as mean \pm standard deviation values for each treatment group. The results were compared between all three treatment groups by one-way analysis of variance (ANOVA) and then the Fisher's test was used for post-hoc analysis. If there were heterogeneous variances among groups, Dunnett's T3 test was used instead. A $P<0.05$ was considered statistically significant. And using SPSS 21.0 software (SPSS Inc., Chicago, IL, USA) for analysis.

\section{RESULTS}

\section{Effects of GLP-2 on Intestinal Tissue Tight Junctions and Tight Junction Proteins}

TEM demonstrated that the surface of the intestinal epithelial cell membrane was normal, the tight junction structure between cells completed, the bridge density high, and the connections dense in the Chow and TPN+GLP-2 groups. The surface of the intestinal epithelial cell membrane was reduced, absent, or lodging, the tight junction structure between cells less complete and partially broken, the bridge particle density decreased, the connections loose, and the gaps between cells widened in the TPN group (Figure 1A).

Immunofluorescence showed that the tight junction proteins ZO-1, occludin, and claudin-1 were located on the surfaces of the villi, and their distributions were continuous and dense in the Chow and TPN+GLP-2 groups. The distributions of the fluorescence of the tight junction proteins $\mathrm{ZO}-1$, occludin, and claudin-1 were discontinuous, partially fragmented, partially displaced into cell interiors, and low in density in the TPN group (Figure 1B).

Compared with the Chow and TPN+GLP-2 groups, Western blot analysis showed that the levels of occludin and claudin-1 proteins in intestinal tissues were significantly decreased in the TPN group $(P<0.05)$ (Figure 1C).

\section{Effects of GLP-2 on Paneth Cells Function in Intestinal Tissue}

To detect the effects of GLP-2 on Paneth cells function in intestinal tissue, we measured the expression of Lysozyme and secretory phospholipase A2 proteins (sPLA2) in intestinal tissue. Compared with the TPN+GLP-2 group, the levels of Lysozyme and sPLA2 were significantly decreased in the TPN group $(P<0.05)$, and no significant difference between the remaining two groups has observed (Figures 2A,B).

\section{Effect of GLP-2 on Intestinal Epithelial Protection and Repair in Intestinal Tissue}

Then, we detected the mRNA levels of Tff1, Tff2, Tff3, Tgfb1, Tgfb2, Tgfb3, Egfr, Hgr and Fgf7, genes of epithelial protection and repair. Compared with the TPN group, the expression of all above genes were increased in the TPN+GLP-2 group $(P<0.05)$, and no 
A
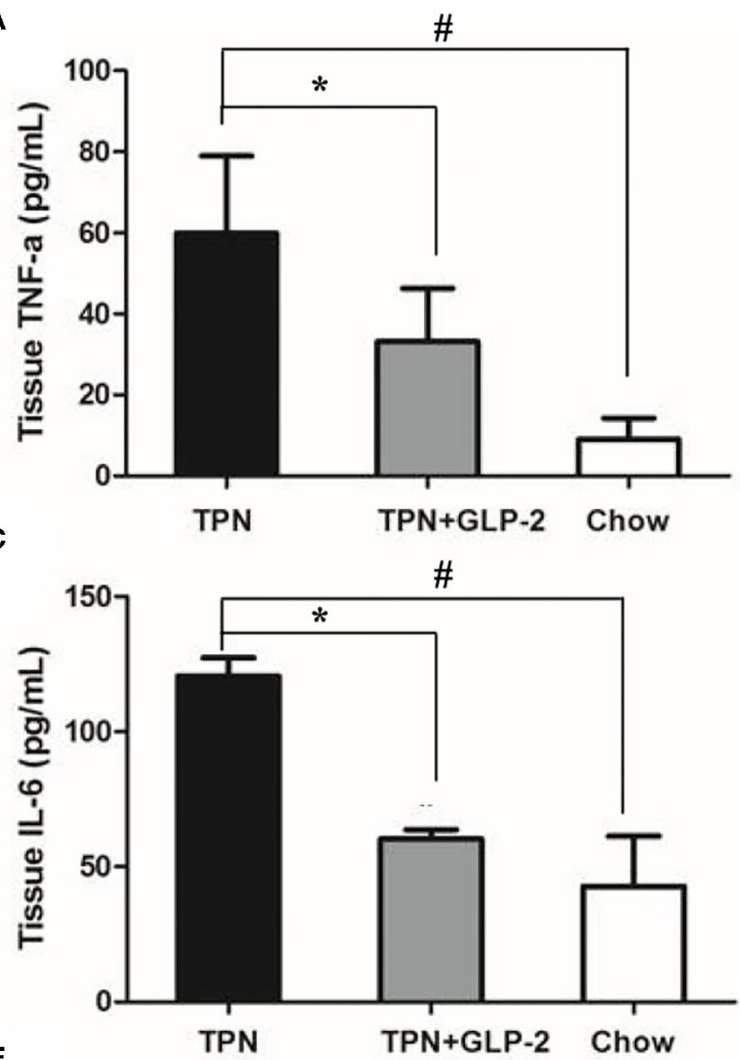

E

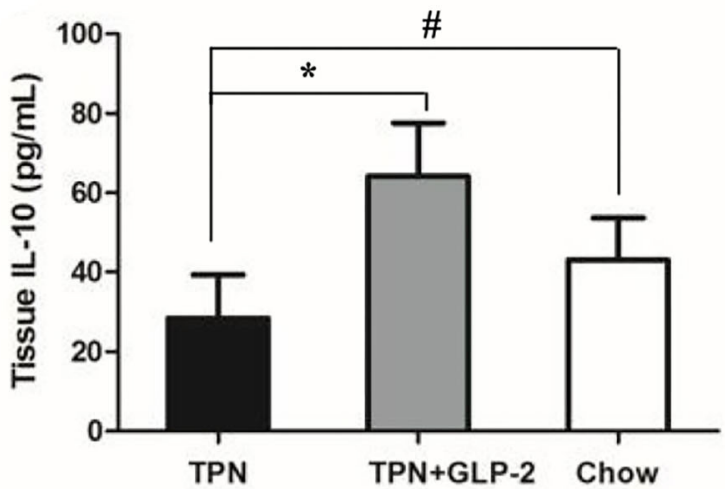

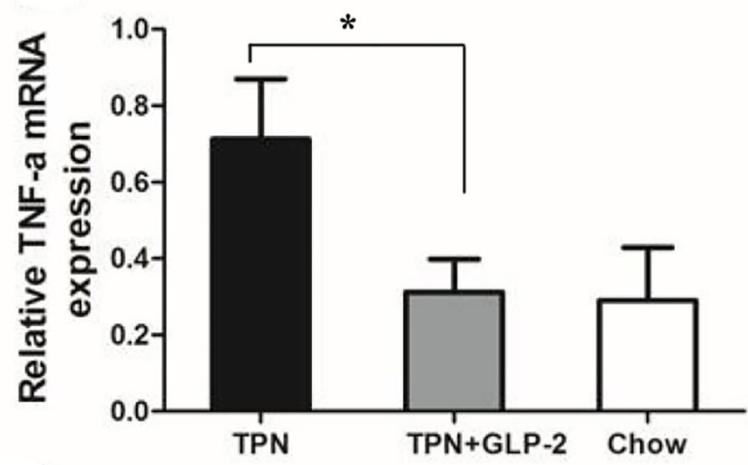

D
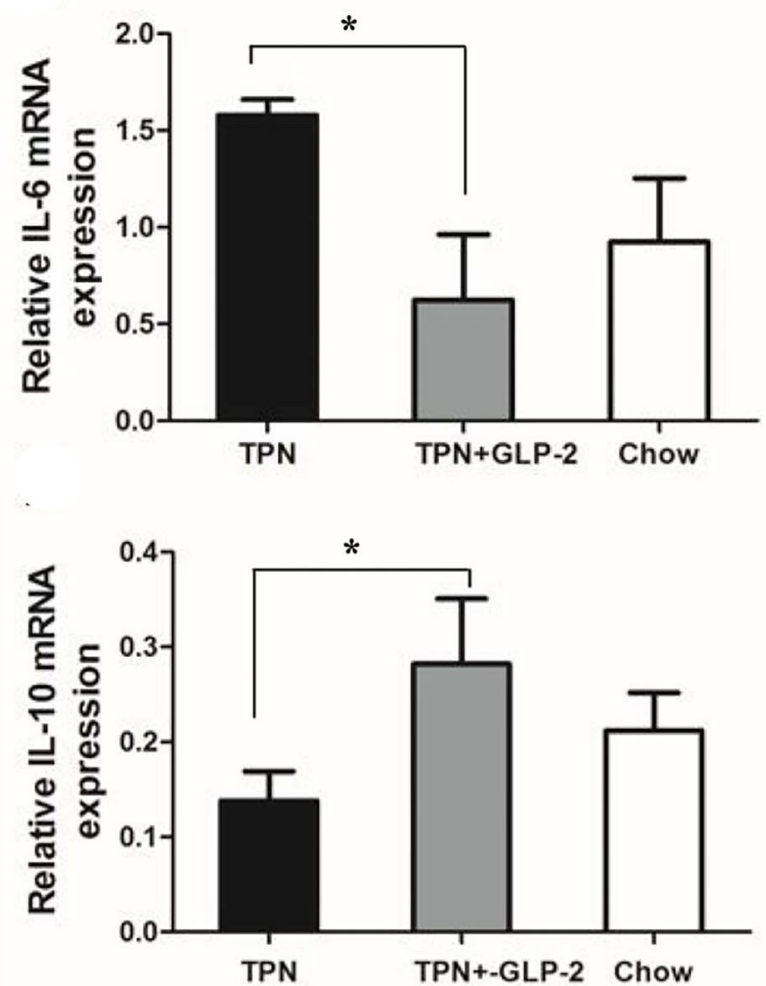

FIGURE 5 | The intestinal inflammatory cytokine levels. (A,B) Expression of TNF- $\alpha$ protein and mRNA. (C,D) Expression of IL-6 protein and mRNA. (E,F) Expression of IL-10 protein and mRNA. Where *indicates $p<0.05$ for TNP vs. TPN+GLP-2 and Chow, and \# indicates $p<0.05$ for TNP vs. Chow.

difference between the TPN+GLP-2 and Chow groups (Figures 3A-I).

\section{Effects of GLP-2 on the Expression of Insulin Like Growth Factor-1 in Intestinal \\ Tissue}

We also measured the level of IGF-1. Mice in the TPN+GLP-2 group have higher mRNA level of IGF-1 than that of mice in the TPN group (All $P<0.05$ ) (Figures 4A,B), as well as the levels in intestinal tissue.
Effects of GLP-2 on Intestinal Inflammatory

\section{Cytokine Levels}

Next, we tested the levels of inflammatory cytokine, such as pro-inflammatory cytokine TNF- $\alpha$ and IL-6, and antiinflammatory factor IL-10. The TPN group had higher protein levels of TNF- $\alpha$ and IL- 6 , and lower levels of IL-10 than that of Chow group. Compared with the TPN groups, the proteins and mRNA levels of TNF- $\alpha$ and IL- 6 were significantly decreased in the TPN+GLP-2 group (all $p<$ 0.05 ), while the proteins and mRNA levels of IL-10 in the intestinal tissues were increased in the TPN+GLP-2 group $(p<0.05)$ (Figures 5A-F). 
A

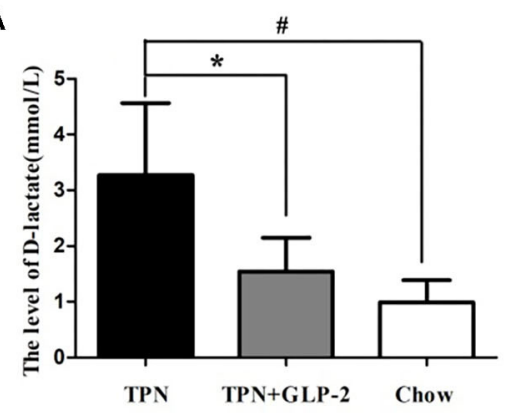

B

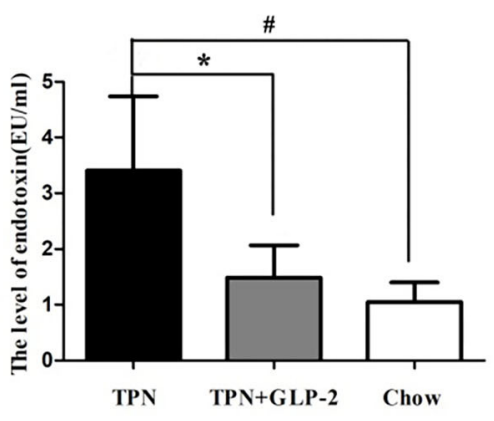

c

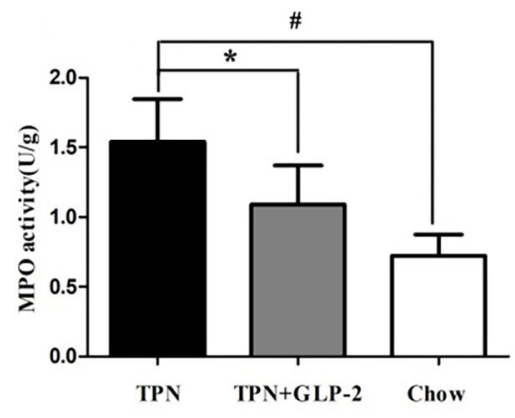

FIGURE 6 | The plasma D-lactate and plasma endotoxin levels and inflammatory infiltration of intestinal tissues. (A) The plasma D-lactate level. (B) The plasma endotoxin level. (C) The intestinal tissue myeloperoxidase (MPO) activity. Where *indicates $p<0.05$ for TNP vs. TPN+GLP-2, and \#indicates $p<0.05$ for TNP vs. Chow.

\section{Effects of GLP-2 on Serum D-lactic Acid and Endotoxin Levels and Inflammatory Infiltration of Intestinal Tissues}

Compared with the Chow group, the serum D-lactic acid and endotoxin levels were significantly increased in the TPN group $(p<0.05)$ (Figures 6A,B). And MPO activity in the intestinal tissues was also increased in the TPN group $(p<$ 0.05) (Figure 6C). After GLP-2 treatment, the D-lactic acid and endotoxin levels, and MPO activity remarkably decreased.

\section{DISCUSSION}

In the case of intestinal function, patients are given priority to oral enteral nutrition. Nevertheless, it is undeniable that in many cases, there is no way for critically ill patients to achieve the goal of energy needs through oral enteral nutrition alone (3). At this time, supplementary parenteral nutrition or total parenteral nutrition is necessary. Parenteral nutrition has been a widely-used therapy for critically ill patients with gastrointestinal dysfunction, and has shown to improve their survival rates and prognosis and to promote rehabilitation outcomes (16). It is worth emphasizing that patients with intestinal integrity even if on PN, must restart minimal enteral feeding as soon as possible (17). The reason is that parenteral nutrition as a non-physiological method of nutrition delivery, has some negative effects on the gastrointestinal tract, including the intensification of intestinal mucosal atrophy, increased intestinal permeability and loss of intestinal integrity. Long term parenteral nutrition also inhibits immune function, which will lead to many clinical complications, such as increased bacterial translocation and sepsis $(18,19)$. Thanks to the normal human intestinal barrier structure (including mechanical, chemical, biological and immunological barriers), the rich bacterial pathogens in the intestinal tract cannot enter into human tissues (20). When the intestinal barrier is destroyed, endotoxin is likely to enter into the circulatory system and cause endotoxemia. Moreover, a significant decrease in the production of mucosa-derived growth factors has been reported in animal models of TPN (21).
Therefore, for patients who rely on TPN, use the exogenous growth factors could be a hopeful approach to promote the growth of intestinal mucosa and improve epithelial barrier function (22). Many researches suggested that GLP-2 and its analogs play a significant role in patients with short bowel syndrome (SBS), including promoting intestinal adaptation, improving the surface area and absorption of the small intestine, and facilitating weaning from parenteral nutrition $(23,24)$. Therefore, it is of clinical significance to explore whether GLP-2 can improve TPN induced intestinal barrier injury by enhancing the intestinal immune function. Our study provides a possible method of patients who rely on IV fluid support to maintain longer intestinal barrier function. Indeed, 5 days of parenteral nutrition intervention is very short compared to treatment time in humans. However, previous studies suggested that the intestinal barrier damage has been severe after 5 days of PN intervention. Therefore, it is of certain significance to detect the influence of GLP-2 on intestinal function of mice after 5 days of during the process of IV infusion mainly consisted of nutritional materials $(25,26)$.

Intestinal innate immunity main consists of antimicrobial peptides produced and released by Paneth cells and mucin secreted by goblet cells (27). Paneth cells are mainly located at the bottom of the intestinal crypts near the stem cells and secrete a large amounts of antibacterial compounds, including lysozyme, sPLA2, RegIII $\gamma$, Angiogenin4, and defensins $(27,28)$. These substances protect the epithelium by preventing bacterial adhesion. In the present study, TPN induced a significant downregulation of the expression of lysozyme and sPLA2 in the small intestinal tissue, which is consistent with previous studies showing that PN can reduce Paneth cell function (29). Our study found that the application of GLP-2 reversed the expression of lysozyme and sPLA2 protein in mouse ileum tissue. These findings support our hypothesis that GLP-2 can improve the Paneth cells immune function during PN via IV infusion.

In this study, we found that the expression of epithelial protection and repair genes were significantly increased in the TPN+GLP-2 group. TPN can reduce the epithelial protection and repair protein synthesis, leading to intestinal barrier damage 
and bacterial translocation. Previous research suggested that PN combined with GLP-2 optimizes various intestinal parameters during post-resection adaptation compared to those observed with PN alone, including small intestine wet weight, villus and microvillus height, crypt cell proliferation, nutrient transporter expression, and nutrient absorption (30). Fgf7 promotes the proliferation of intestinal epithelial cells, which is also able to decrease the apoptosis induced by parenteral nutrition $(31,32)$. The administration of Fgf7 can reverse the current decline of anti-apoptotic Bcl-2 during TPN (30). The previous study found that the interactions of Fgf7 with the trefoil factor family promote intestinal mucosa repair (33). Our findings identify that GLP-2 could repair intestinal mucosal damage during $\mathrm{PN}$ via IV infusion.

In this study, the level of insulin like growth factor-1 protein and mRNA expression were significantly increased in the TPN+GLP-2 group. Previous studies have shown that IGF-1 can act on the intestinal epithelium through the endocrine effect of circulating IGF-1 mainly derived from hepatocytes and the paracrine effect of locally expression of IGF-1 synthesized by the intestinal mesenchyme (34). Growth hormone (GH), calorie and protein intake, and insulin have a positive regulatory effect on circulating IGF-1 circulation. Except in the case of extreme GH deficiency or excess, local intestinal IGF-1 is less regulated by GH but is positively regulated by luminal nutrients. There is some convincing evidence support that IGF-1 is an essential mediator of these effects on GLP-2, which stems from recent observations that GLP-2 cannot effectively increase the small intestine mass, crypt depth or villus height in mice with a targeted disruption of IGF-1 alleles (34) and mice with inducible deletion of IGF-1R specifically in intestinal epithelial cells (IECs) (35). Our research results are consistent with a previous study showing that GLP2 enhances intestinal IGF-1 expression and also are required for small intestinal growth in response to GLP-2 (36). These findings confirm that IGF-1 is an important mediator of the intestinal tropic effect of GLP-2.

Numerous studies have documented damage to intestinal barrier function by pro-inflammatory cytokines in both intestinal epithelial and intestinal endothelial cells $(37,38)$. In contrast, some anti-inflammatory cytokines (such as: IL-10) have been shown to attenuate the intestinal epithelial barrier damage caused by IFN- $\gamma$. Therefore, it can be noticed that inflammatory cytokines can regulate the integrity of intestinal mucosal barrier. Previous studies found that TPN application reduced IL-4 and IL-10 levels in the intestine tissue, while increasing IFN- $\gamma$ levels, which inhibited the production of IgA (39). In this study, we found that the proteins and mRNAs expression of TNF- $\alpha$ and IL-6 and MPO activity in the intestinal tissues were significantly increased in the TPN group, but IL-10 levels were decreased. GLP-2 could reverse the above changes in inflammatory factor expression levels. These results confirmed our hypothesis that GLP2 alleviates gut inflammation during on subjects whose nutritional needs are mainly rely upon IV infusion.

D-lactic acid is the metabolic end product of intestinal microorganisms such as Lactic Acid Bacteria and Escherichia coli (40). When the permeability of the intestinal mucosa increases, the D-lactic acid produced by the bacteria in the intestinal cavity enters the blood through the damaged mucous membrane, thereby increasing the plasma D-lactic acid concentration. Therefore, the D-lactic acid content in the peripheral blood can reflect the permeability of the intestinal mucosal barrier and the degree of impairment of intestinal mucosal barrier function. In this study, we also observed that both the Dlactic acid and serum endotoxin levels in the TPN group were higher than those in the TPN+GLP-2 group, which further demonstrated that GLP-2 could restored damage to the intestinal mucosal barrier during parenteral nutrition. Our results suggest that GLP-2 can alleviate gut inflammation and improve intestinal barrier function in line with previous studies $(41,42)$.

In conclusion, GLP-2 alleviated gut inflammation and improved enteric Paneth cells immune responses during $\mathrm{PN}$ via IV infusion in mice with a central catheter, possibly by improving the functioning of epithelial protection and repair and reducing mucosal inflammatory responses.

\section{DATA AVAILABILITY STATEMENT}

The raw data supporting the conclusions of this article will be made available by the authors, without undue reservation.

\section{ETHICS STATEMENT}

The animal study was reviewed and approved by the animal Ethics Committee of Jinling Hospital (Ethical approval number: 2018NZGKJ-034).

\section{AUTHOR CONTRIBUTIONS}

XW developed the overall research plan and oversaw the study. GD and QL designed and conducted of the study, and completed the writing of the manuscript. XG, YZ, HZ, and JB helped the data collection and processing. The authors accept full responsibility for the design and conduct of the study, have access to the data, and controlled the decision to publish. All authors contributed to the article and approved the submitted version.

\section{FUNDING}

This study was financially supported in part by National Natural Science Foundation of China (81770531) and Science Foundation of outstanding youth in Jiangsu (BK20170009).

\section{SUPPLEMENTARY MATERIAL}

The Supplementary Material for this article can be found online at: https://www.frontiersin.org/articles/10.3389/fnut.2021. 688715/full\#supplementary-material 


\section{REFERENCES}

1. Denton C, Price A, Friend J, Manithody C, Blomenkamp K, Westrich M, et al. Role of the gut-liver axis in driving parenteral nutrition-associated injury. Children. (2018) 5:136. doi: 10.3390/children5100136

2. Madnawat H, Welu AL, Gilbert EJ, Taylor DB, Jain S, Manithody C, et al. Mechanisms of parenteral nutrition-associated liver and gut injury. Nutr Clin Pract. (2020) 35:63-71. doi: 10.1002/ncp.10461

3. Pironi L, Corcos O, Forbes A, Holst M, Joly F, Jonkers C, et al. Intestinal failure in adults: recommendations from the ESPEN expert groups. Clin Nutr. (2018) 37:1798-809. doi: 10.1016/j.clnu.2018.07.036

4. Dudrick SJ, Palesty JA. Historical highlights of the development of total parenteral nutrition. Surg Clin North Am. (2011) 91:693-717. doi: 10.1016/j.suc.2011.02.009

5. Costakos DT. Of lobsters, electronic medical records, and neonatal total parenteral nutrition. Pediatrics. (2006) 117:e32832. doi: 10.1542/peds.2005-1350

6. Boullata JI. Overview of the parenteral nutrition use process. JPEN $J$ Parenter Enteral Nutr. (2012) 36(Suppl. 2):S10-3. doi: 10.1177/01486071114 33624

7. Ring LL, Nerup N, Jeppesen PB, Svendsen LB, Achiam MP. Glucagon like peptide-2 and neoplasia; a systematic review. Expert Rev Gastroenterol Hepatol. (2018) 12:257-64. doi: 10.1080/17474124.2018.14 17032

8. Nerup N, Ambrus R, Lindhe J, Achiam MP, Jeppesen PB, Svendsen LB. The effect of glucagon-like peptide-1 and glucagon-like peptide2 on microcirculation: a systematic review. Microcirculation. (2019) 26:e12367. doi: 10.1111/micc.12367

9. Chen X, Zhao HX, Fu XS, Li CP, Zhong XL. Glucagonlike peptide 2 protects intestinal barrier in severe acute pancreatitis through regulating intestinal epithelial cell proliferation and apoptosis. Pancreas. (2012) 41:10805. doi: 10.1097/MPA.0b013e31824966b0

10. Lei Q, Bi J, Chen H, Tian F, Gao X, Li N, et al. Glucagon-like peptide-2 improves intestinal immune function and diminishes bacterial translocation in a mouse model of parenteral nutrition. Nutr Res. (2018) 49:5666. doi: 10.1016/j.nutres.2017.10.007

11. Carroll RE, Benedetti E, Schowalter JP, Buchman AL. Management and complications of short bowel syndrome: an updated review. Curr Gastroenterol Rep. (2016) 18:40. doi: 10.1007/s11894-016-0511-3

12. Billiauws L, Joly F. Emerging treatments for short bowel syndrome in adult patients. Expert Rev Gastroenterol Hepatol. (2019) 13:241-6. doi: 10.1080/17474124.2019.1 569514

13. Zhang T, Shi L, Xu Y, Li Y, Li S, Guan B, et al. Purified PEGylated human glucagon-like peptide-2 reduces the severity of irradiation-induced acute radiation enteritis in rats. J Radiat Res. (2019) 60:7-16. doi: 10.1093/jrr/rry076

14. Lei Q, Bi J, Wang X, Jiang T, Wu C, Tian F, et al. GLP-2 prevents intestinal mucosal atrophy and improves tissue antioxidant capacity in a mouse model of total parenteral nutrition. Nutrients. (2016) 8:33. doi: 10.3390/nu80 10033

15. Wan X, Bi J, Gao X, Tian F, Wang X, Li N, et al. Partial enteral nutrition preserves elements of gut barrier function, including innate immunity, Intestinal Alkaline Phosphatase (IAP) level, and intestinal microbiota in mice. Nutrients. (2015) 7:6294-312. doi: 10.3390/nu70 85288

16. Mogensen KM, Robinson MK, Casey JD, Gunasekera NS, Moromizato $\mathrm{T}$, Rawn JD, et al. Nutritional status and mortality in the critically ill. Crit Care Med. (2015) 43:2605-15. doi: 10.1097/CCM.00000000000 01306

17. Reintam Blaser A, Starkopf J, Alhazzani W, Berger MM, Casaer MP, Deane AM, et al. Early enteral nutrition in critically ill patients: ESICM clinical practice guidelines. Intensive Care Med. (2017) 43:38098. doi: 10.1007/s00134-016-4665-0

18. Feng Y, Demehri FR, Xiao W, Tsai YH, Jones JC, Brindley CD, et al. Interdependency of EGF and GLP-2 signaling in attenuating mucosal atrophy in a mouse model of parenteral nutrition. Cell Mol Gastroenterol Hepatol. (2017) 3:447-68. doi: 10.1016/j.jcmgh.2016.12.005
19. Heneghan AF, Pierre JF, Tandee K, Shanmuganayagam D, Wang X, Reed JD, et al. Parenteral nutrition decreases paneth cell function and intestinal bactericidal activity while increasing susceptibility to bacterial enteroinvasion. JPEN J Parenter Enteral Nutr. (2014) 38:817-24. doi: 10.1177/01486071134 97514

20. Chen J, Dong JT, Li XJ, Gu Y, Cheng ZJ, Cai YK. Glucagon-like peptide2 protects impaired intestinal mucosal barriers in obstructive jaundice rats. World J Gastroenterol. (2015) 21:484-90. doi: 10.3748/wjg.v21.i2.484

21. Brinkman AS, Murali SG, Hitt S, Solverson PM, Holst JJ, Ney DM. Enteral nutrients potentiate glucagon-like peptide-2 action and reduce dependence on parenteral nutrition in a rat model of human intestinal failure. Am J Physiol Gastrointest Liver Physiol. (2012) 303:G610-22. doi: 10.1152/ajpgi.00184.2012

22. Warner BW. The pathogenesis of resection-associated intestinal adaptation. Cell Mol Gastroenterol Hepatol. (2016) 2:42938. doi: 10.1016/j.jcmgh.2016.05.001

23. Sigalet DL, Brindle M, Boctor D, Casey L, Dicken B, Butterworth S, et al. Safety and dosing study of glucagon-like peptide 2 in children with intestinal failure. JPEN J Parenter Enteral Nutr. (2017) 41:84452. doi: $10.1177 / 0148607115609566$

24. Pironi L, Arends J, Bozzetti F, Cuerda C, Gillanders L, Jeppesen PB, et al. ESPEN guidelines on chronic intestinal failure in adults. Clin Nutr. (2016) 35:247-307. doi: 10.1016/j.clnu.2016.01.020

25. Ikeda S, Zarzaur BL, Johnson CD, Fukatsu K, Kudsk KA. Total parenteral nutrition supplementation with glutamine improves survival after gut ischemia/reperfusion. JPEN J Parenter Enteral Nutr. (2002) 26:169-73. doi: 10.1177/0148607102026003169 PMID: 12005457

26. DeWitt RC, Wu Y, Renegar KB, King BK, Li J, Kudsk KA. Bombesin recovers gut-associated lymphoid tissue and preserves immunity to bacterial pneumonia in mice receiving total parenteral nutrition. Ann Surg. (2000) 231:1-8. doi: 10.1097/00000658-200001000-00001

27. Heneghan AF, Pierre JF, Kudsk KA. JAK-STAT and intestinal mucosal immunology. JAKSTAT. (2013) 2:e25530. doi: 10.4161/jkst.25530

28. Porter EM, Bevins CL, Ghosh D, Ganz T. The multifaceted Paneth cell. Cell Mol Life Sci. (2002) 59:156-70. doi: 10.1007/s00018-002-8412-z

29. Heneghan AF, Pierre JF, Gosain A, Kudsk KA. IL-25 improves luminal innate immunity and barrier function during parenteral nutrition. Ann Surg. (2014) 259:394-400. doi: 10.1097/SLA.0b013e318284f510

30. Tappenden KA. Intestinal adaptation following resection. JPEN J Parenter Enteral Nutr. (2014) 38(Suppl. 1):S23-31. doi: 10.1177/0148607114525210

31. Walters JR. Cell and molecular biology of the small intestine: new insights into differentiation, growth and repair. Curr Opin Gastroenterol. (2004) 20:706. doi: 10.1097/00001574-200403000-00004

32. Wildhaber BE, Yang H, Teitelbaum DH. Total parenteral nutrition-induced apoptosis in mouse intestinal epithelium: modulation by keratinocyte growth factor. J Surg Res. (2003) 112:144-51. doi: 10.1016/s0022-4804(03)00160-4

33. Fernandez-Estivariz C, Gu LH, Gu L, Jonas CR, Wallace TM, Pascal RR, et al. Trefoil peptide expression and goblet cell number in rat intestine: effects of KGF and fasting-refeeding. Am J Physiol Regul Integr Comp Physiol. (2003) 284:R564-73. doi: 10.1152/ajpregu.00428.2002

34. Bortvedt SF, Lund PK. Insulin-like growth factor 1: common mediator of multiple enterotrophic hormones and growth factors. Curr Opin Gastroenterol. (2012) 28:89-98. doi: 10.1097/MOG.0b013e32835004c6

35. Rowland KJ, Trivedi S, Lee D, Wan K, Kulkarni RN, Holzenberger M, et al. Loss of glucagon-like peptide-2-induced proliferation following intestinal epithelial insulin-like growth factor-1-receptor deletion. Gastroenterology. (2011) 141:2166-75.e7. doi: 10.1053/j.gastro.2011.09.014

36. Dubé PE, Forse CL, Bahrami J, Brubaker PL. The essential role of insulin-like growth factor-1 in the intestinal tropic effects of glucagon-like peptide-2 in mice. Gastroenterology. (2006) 131:589-605. doi: 10.1053/j.gastro.2006.05.055

37. Walsh SV, Hopkins AM, Nusrat A. Modulation of tight junction structure and function by cytokines. Adv Drug Deliv Rev. (2000) 41:30313. doi: 10.1016/s0169-409x(00)00048-x

38. Omonijo FA, Liu S, Hui Q, Zhang H, Lahaye L, Bodin JC, et al Thymol improves barrier function and attenuates inflammatory responses in porcine intestinal epithelial cells during Lipopolysaccharide (LPS)-induced inflammation. J Agric Food Chem. (2019) 67:61524. doi: 10.1021 /acs.jafc. 8 b05480 
39. Anastasilakis CD, Ioannidis O, Gkiomisi AI, Botsios D. Artificial nutrition and intestinal mucosal barrier functionality. Digestion. (2013) 88:193208. doi: $10.1159 / 000353603$

40. Bell V, Ferrão J, Pimentel L, Pintado M, Fernandes T. One health, fermented foods, and gut microbiota. Foods. (2018) 7:195. doi: 10.3390/foods7120195

41. Brubaker PL. Glucagon-like peptide- 2 and the regulation of intestinal growth and function. Compr Physiol. (2018) 8:1185-210. doi: 10.1002/cphy.c170055

42. Topaloglu N, Küçük A, Yildirim S, Tekin M, Erdem H, Deniz M. Glucagonlike peptide- 2 exhibits protective effect on hepatic ischemia-reperfusion injury in rats. Front Med. (2015) 9:368-73. doi: 10.1007/s11684-015-0403-1

Conflict of Interest: The authors declare that the research was conducted in the absence of any commercial or financial relationships that could be construed as a potential conflict of interest.
Publisher's Note: All claims expressed in this article are solely those of the authors and do not necessarily represent those of their affiliated organizations, or those of the publisher, the editors and the reviewers. Any product that may be evaluated in this article, or claim that may be made by its manufacturer, is not guaranteed or endorsed by the publisher.

Copyright $\odot 2021$ Deng, Lei, Gao, Zhang, Zheng, Bi and Wang. This is an openaccess article distributed under the terms of the Creative Commons Attribution License (CC BY). The use, distribution or reproduction in other forums is permitted, provided the original author(s) and the copyright owner(s) are credited and that the original publication in this journal is cited, in accordance with accepted academic practice. No use, distribution or reproduction is permitted which does not comply with these terms. 